Research Paper

\title{
Water extract of tendril of Cucurbita Moschata Duch. suppresses RANKL-induced osteoclastogenesis by down-regulating $\mathrm{P} 38$ and ERK signaling
}

\author{
Joo-Hee Choi1,2, Ah-Ra Jang1, Ha-Na Jeong33, Kiok Kim³, Young-Min Kim³, Jeong-Yong Cho3, Jong-Hwan \\ Park $1^{\bowtie}$ \\ 1. Laboratory Animal Medicine, College of Veterinary Medicine and BK 21 PLUS Project Team, Chonnam National University, Gwangju, Republic of Korea. \\ 2. Laboratory Animal Center, Daegu-Gyeongbuk Medical Innovation Foundation, Daegu, Republic of Korea. \\ 3. Department of Food Science \& Technology, Chonnam National University, Gwangju, Republic of Korea. \\ $\square$ Corresponding author: Jong-Hwan Park, DVM, Ph.D; Laboratory Animal Medicine, College of Veterinary Medicine and Brain Korea 21 PLUS Project Team, \\ Chonnam National University 77 Yongbong-ro, Buk-gu, Gwangju 61186, Republic of Korea. Tel: +82-62-530-2834; E-mail: jonpark@jnu.ac.kr.
}

(1) The author(s). This is an open access article distributed under the terms of the Creative Commons Attribution License (https://creativecommons.org/licenses/by/4.0/). See http://ivyspring.com/terms for full terms and conditions.

Received: 2019.08.25; Accepted: 2020.02.12; Published: 2020.02.24

\begin{abstract}
Background: Pumpkin (Curcubita sp.) is a natural product that is commonly used in folk medicine. However, the inhibitory effect and molecular mechanisms of tendril of Cucurbita Moschata Duch. (TCMD) on osteoclast differentiation have yet to be clearly elucidated. Thus, the present study aimed to investigate the effect and underlying mechanism of water extract of TCMD on osteoclast differentiation.
\end{abstract}

Methods: Bone marrow-derived macrophages (BMDMs), osteoclast precursors, were cultured with macrophage colony stimulating factor (M-CSF) $30 \mathrm{ng} / \mathrm{ml}$ and receptor activator of nuclear factor-kappa B ligand (RANKL) $100 \mathrm{ng} / \mathrm{ml}$ for four days. We investigated the effect of TCMD on RANKL-induced osteoclast differentiation, tartrate-resistant acid phosphatase (TRAP) staining, F-actin ring formation, and bone resorption assay. RANKL signaling pathways were determined through Western blotting, and osteoclast differentiation marker genes were confirmed by Real-time PCR.

Results: TCMD inhibited the RANKL-induced osteoclast differentiation in a dose-dependent manner without cytotoxicity. Further, F-actin ring formation and bone resorption were reduced by TCMD in RANKL-treated BMDMs. In addition, TCMD decreased the phosphorylation of p38 and ERK as well as the expression of osteoclast-related genes in BMDMs treated with RANKL.

Conclusion: These findings suggest that TCMD may have preventive and therapeutic effects for destructive bone diseases.

Key words: Osteoclast differentiation, RANKL, Tendril of Cucurbita Moschata Duch. (TCMD)

\section{Introduction}

Bone is a dynamic organ that maintains its structure and function by replacing the old and damaged bone with new bone via bone remodeling. The bone remodeling cycle involves a balanced interaction between osteoclastic bone resorption and osteoblastic bone formation [1]. Osteoclasts, multinucleated bone-resorbing cells, are derived from the hematopoietic precursor cells of monocyte/macrophage lineage at various stages, including proliferation, migration, fusion, and activation [2]. Osteoclastogenesis is an osteoblast-dependent process that is regulated by two critical cytokines: the macrophage colony-stimulating factor (M-CSF) and receptor activator of nuclear 
factor-kappa B (NF-kB) ligand (RANKL) [3]. M-CSF plays an important role in the survival and proliferation of precursors of the monocyte/ macrophage lineage. RANKL is secreted by osteoblasts and binds to its receptor RANK to differentiate osteoclast precursors into osteoclasts $[4,5]$. The binding of RANKL to RANK activates the mitogen-activated protein kinase (MAPK) pathway and NF- $\mathrm{kB}$, and the downstream factors including the nuclear factor of activated T cells c1 (NFATc1), which is the master regulator of osteoclastogenesis [6,7]. The activation of these signal molecules results in the differentiation and activation of osteoclasts, which play a role in bone resorption and actin ring formation [8]. Abnormal osteoclastogenesis or osteoclast activation leads to an imbalance in bone remodeling, which predominantly causes osteoporosis, rheumatoid arthritis, and periodontitis. In attempts to develop therapeutic reagents to treat bone diseases caused by deregulated osteoclastogenesis, the signaling molecules and transcription factors under RANKL signaling axis for osteoclastogenesis have been extensively investigated.

Natural plant products are attracting increasing attention for their efficacy in the prevention and treatment of various metabolic diseases. The pumpkin plant (Curcubita sp.) is a ubiquitous edible vine. The species of pumpkins that are commonly available include Cucurbita pepo (most common), Cucurbita maxima, Cucurbita stilbo, and Cucurbita Moschata [9]. There is evidence showing that Curcubita sp. have beneficial effects on a variety of diseases. For example, fermented Cucurbita moschata extract has been shown to have anti-obesity potential by suppressing body weight gain by regulating adipogenic transcriptional factors [10]. Additionally, pumpkin seed extract has been reported to have estrogenic properties in a rat model as well as the potential to overcome symptoms caused by estrogen deficiency [11]. Cucurbita Moschata Duch. has long been considered a health food in many countries such as Mexico, India, China, Brazil, and Korea [12]. Cucurbita Moschata Duch. flesh and seeds are nutritionally rich due to the proteins and antioxidant vitamins such as carotenoids and tocopherols [13]. Dehydrodiconiferyl alcohol (DHCA), a lignan originally isolated from Cucurbita moschata, is believed to be a phytoestrogen based on its structure and is also thought to prevent OVX-induced osteoporosis by inhibiting osteoclastogenesis [14]. However, the inhibitory potential and molecular mechanisms of tendril of Cucurbita Moschata Duch. (TCMD) extract on RANKL-induced osteoclast differentiation have yet to be elucidated.

In this study, we investigated the inhibitory effect of TCMD extract on RANKL-induced osteoclast differentiation, provided molecular mechanisms explaining its inhibitory activity, and suggested possibilities for the use of TCMD as a traditional medicine against bone diseases.

\section{Materials and Methods}

\section{Preparation of pumpkin tendril water extract}

The dried tendril of pumpkin (Cucurbita Moschata Duch.) was purchased at the Jjanggu-oppa agricultural market (Jinju, Gyeongsangnam-do, Korea). The pumpkin tendril was identified by Prof. Kang-Mo Ku, Department of Horticulture Science, Chonnam National University. A voucher sample (no. JNUCM20180807) was deposited in the herbarium of the laboratory. The pumpkin tendrils (200 g) were washed well in purified water and then placed in the extractor (DW290, Daewoongbio, Seoul, Korea). After the addition of $1 \mathrm{~L}$ of distilled water, it was extracted at $90{ }^{\circ} \mathrm{C}$ for $4 \mathrm{~h}$. The extracted samples were filtered through a glass fiber filter on a Buchner funnel and concentrated through vacuum evaporation (N1110 EYELA, Tokyo, Japan). The collected samples were stored at $-20{ }^{\circ} \mathrm{C}$ for $48 \mathrm{~h}$ in a freezer (RB 603GMWP, LG, Seoul, Korea) and lyophilized (TFD8503 Ilshin Lab Co., Ltd, Seoul, Korea).

\section{Osteoclast differentiation and TRAP staining}

Mouse bone marrow cells (BMCs) were isolated from the femurs of six-to-eight-week-old male C57 BL/6 (KOATECH, Gyunggi-Do, Korea) [15]. After lysing the red blood cells, the cells were incubated for three days in $\mathrm{CO}_{2}$ incubator in a-minimal essential medium (MEM) (Gibco) with 10\% fetal bovine serum (Corning) containing $30 \mathrm{ng} / \mathrm{ml}$ M-CSF. The attached cells (BMDMs, bone marrow-derived macrophages) were used as osteoclast precursors by removing floating cells. For generation of osteoclasts, the BMDMs were seeded in 12-well plates with M-CSF plus RANKL for four days. Osteoclast formation was determined through tartrate-resistant acid phosphatase (TRAP) staining. For TRAP staining, the cells were fixed with a fixative solution and stained with a commercial kit (SIGMA) according to the manufacturer's instructions. After staining, TRAP-positive multinucleated cells (TRAP+MNCs) containing more than three nuclei were counted under a light microscope. All the animal experiments for primary bone marrow cells were performed according to the Institutional Animal Care and Use Committee (IACUC) of Chonnam National University (approval number: CNU IACUC-YB-2016-34).

\section{Cell viability assay}

First, BMDMs were seeded in a 96-well plate 
with M-CSF (30 ng/ml) and treated with different concentrations of $\operatorname{TCMD}(0.125,0.25,0.5$, and 1 $\mathrm{mg} / \mathrm{ml}$ ). After $24 \mathrm{~h}$, the EZ-Cytox (Daeillab service, Seoul, $\mathrm{Kr})$ solution $(10 \mu \mathrm{l})$ was added to each well and the plate was incubated for $3 \mathrm{~h}$ in a $\mathrm{CO}_{2}$ incubator. An ELx808 microplate spectrophotometer reader (BioTek, Winooski, VT, USA) was used to measure the absorbance at $450 \mathrm{~nm}$.

\section{Western blotting}

For Western blotting, BMDMs were seeded in 12-well plate and incubated overnight. These cells were treated with TCMD in the presence or absence of RANKL. At the indicated time point after treatment, cells were lysed in a buffer containing 1\% NP-40, 50 $\mathrm{mM}$ Tris (pH 7.4), $250 \mathrm{mM} \mathrm{NaCl}, 5 \mathrm{mM}$ EDTA, $50 \mathrm{mM}$ $\mathrm{NaF}, 1 \mathrm{Mm} \mathrm{Na}_{3} \mathrm{VO}_{4}, 0.02 \% \mathrm{NaN}_{3}, 2 \mathrm{mM}$ dithiothreitol plus protease inhibitor (Roche, Mannheim, Germany) and phosphatase inhibitor (Sigma-Aldrich). Whole cell lysates were separated using SDS-PAGE and transferred onto nitrocellulose membranes The membranes were probed with primary antibodies against IkBa, phospho-form of p38, ERK, JNK, and p65 (Cell Signaling Technology, Danvers, MA, USA). A primary antibody for $\beta$-actin was obtained from Santa Cruz. The membranes were then washed with TBST and incubated with HRP-conjugated secondary antibodies (Santa Cruz, CA, USA) for $2 \mathrm{~h}$ at room temperature. The bands were visualized using Clarity Western ECL Substrate (Bio-Rad, Hercules, CA, USA) on a luminescent image analyzer (Amersham Imager 600, GE Healthcare, UK).

\section{Real-time quantitative polymerase chain reaction (qPCR)}

Total RNA was isolated from osteoclasts using easy-Blue (iNtRON Biotechnology, Seongnam, Korea) and complementary DNA was synthesized from $1 \mu \mathrm{g}$ RNA using an RT Premix reverse transcription system (AccuPower, Seoul, Korea). Then, qPCR was performed using cDNA as a template with Power SYBR Green (Applied Biosystems, Warrington, UK). $\beta$-actin was used for normalization. qPCR was performed for 40 cycles at $95{ }^{\circ} \mathrm{C}$ for $10 \mathrm{~s}, 60{ }^{\circ} \mathrm{C}$ for 60 s using the Rotor-Gene Q real-time PCR system (Qiagen, Hilden, Germany).

The following primers were used: TRAP, 5'-CTG GAGTGCACGATGCCAGCGACA-3' and 5'-TCCGTG CTCGGCGATGGACCAGA-3'; DC-STAMP, 5'-CCAA GGAGTCGTCCATGATT-3' and 5'-GGCTGCTTTGAT CGTTTCTC-3'; Cathepsin K, 5'-GGCCAACTCAAG AAGAAAAC-3' and 5'-GTGCTTGCTTCCCTTCT GG-3'; NFATc1, 5'-CTCGAAAGACAGTGGAGC AT-3' and 5'-CGGCTGCCTTCCGTCTCATAG-3'; and $\beta$-actin, 5'-AGGCCCAGAGCAAGAGAG-3' and
5'-TCAACATGATCTGGGTCATC-3'.

\section{Bone resorption assay}

To observe osteoclast-mediated bone resorption in vitro [16], BMDMs were cultured for four days with M-CSF and RANKL in the presence or absence of TCMD on an Osteo assay surface 24-well plate (Corning Inc., NY). The medium was aspirated from the resorption assay plate and $20 \%$ sodium dodecyl sulfate (SDS) was added for $15 \mathrm{~min}$ to remove the cells. The plate was washed 3 times with distilled water and subsequent air drying. The areas absorbed on the discs were observed using a microscope (Eclipse Ni-U, Nikon, Japan).

\section{F-actin ring formation staining}

In order to evaluate the formation of actin ring in vitro [16], BMDMs differentiated in four days with RANKL and TCMD on a cover glass. The cells were fixed with $4 \%$ paraformaldehyde and permeabilized with $0.2 \%$ Triton X-100/PBS for 10 mins, then stained with $0.1 \%$ Alexa Fluor 594-phalloidin (Invitrogen) and with DAPI (Invitrogen). The images were obtained using a fluorescence microscope (Korea lab tech, Korea).

\section{Statistical analysis}

All statistical calculations were performed using GraphPad Prism version 5.01 (GraphPad, La Jolla, CA, USA). One-way ANOVA followed by Tukey's post-hoc test was used to assess the differences between specific groups. A result was considered statistically significant if the $P$-value was less than 0.05 .

\section{Results}

\section{Water extract of tendril of C. Moschata Duch. (TCMD) suppresses RANKL-induced osteoclast differentiation in BMDMs}

In order to examine the effects of TCMD on RANKL-induced osteoclast differentiation, BMDMs were treated with various concentrations of the water extract of TCMD in the presence of M-CSF and RANKL. Treatment with RANKL effectively differentiated the BMDMs of the control group into TRAP-positive mature multinucleated osteoclasts, as shown by TRAP staining. By contrast, the water extract of TCMD decreased the formation of osteoclast from BMDMs in a dose-dependent manner (Fig. 1A and B). To confirm whether the inhibitory effect of TCMD can be attributed to the toxicity of this extract, we examined cell viability assays in the culture after 24h. The results show that, compared to the control treatment, TCMD had no cytotoxic effect on BMDMs at concentrations below $1 \mathrm{mg} / \mathrm{ml}$ (Fig. 2). 
Water extract of tendril of $C$. Moschata Duch. (TCMD) inhibits the formation of F-actin ring and bone resorption activity of mature osteoclasts

As TCMD suppressed osteoclast differentiation, we examined whether the extract of TCMD has the potential to inhibit the function of osteoclasts derived from BMDMs. In order to examine the effect of TCMD on osteoclastic bone resorption, BMDMs were cultured on an Osteo assay surface plate with various concentrations of TCMD in the presence or absence of RANKL for three days, and the resorbed area was observed under a light microscope. Then, the number and area of resorption pits remarkably decreased in the TCMD-treated group (Fig. 3A). The F-actin ring in osteoclasts is essential for bone resorption. Therefore, we visualized the actin cytoskeleton of differentiated BMDMs through rhodamine-phalloidin staining. Fluorescence microscopy revealed that the F-actin ring structure was abolished by TCMD treatment in the RANKL-treated cells (Fig. 3B).

Water extract of tendril of C. Moschata Duch. (TCMD) down-regulates RANKL-induced NF-KB and MAPKs activation in vitro

RANKL binding to its receptor, RANK, activates MAP kinases (ERK, JNK, and p38) signaling pathways, transferring RANKL signaling to the related transcriptional factors for osteoclast differentiation [17]. In order to investigate the molecular mechanism by which TCMD suppressed RANKL-induced osteoclastogenesis, we examined the inhibitory effects of TCMD on RANKL-induced early signaling events, such as NF-kB and MAPKs signaling pathways. First, BMDMs were pretreated with TCMD for $2 \mathrm{~h}$ and subsequently simulated with RANKL in the presence of TCMD for the indicated time points. The stimulation of BMDMs with RANKL increased the phosphorylation levels of ERK, p38, JNK, and p65, as well as the degradation of IKBa. However, pretreatment with TCMD significantly decreased the phosphorylation levels of p38 and ERK, not JNK (Fig. 4). By contrast, TCMD did not block the RANKL-induced degradation of IKBa protein, suggesting that it could not inhibit RANKL-induced NF-KB activation (Fig. 4).

\section{Water extract of tendril of C. Moschata Duch. (TCMD) suppresses RANKL-induced gene expression of osteoclastogenesis-related factors.}

We further investigated whether TCMD influences the RANKL-induced gene expression such as TRAP, osteoclast-associated receptor (OSCAR), Cathepsin K, dendritic cell-specific trans-membrane protein (DC STAMP), and NFATc1. The results of real-time PCR analysis showed that the expressions of all of the tested genes were upregulated by RANKL, but that they were mostly abolished by TCMD treatment (Fig. 5).

a

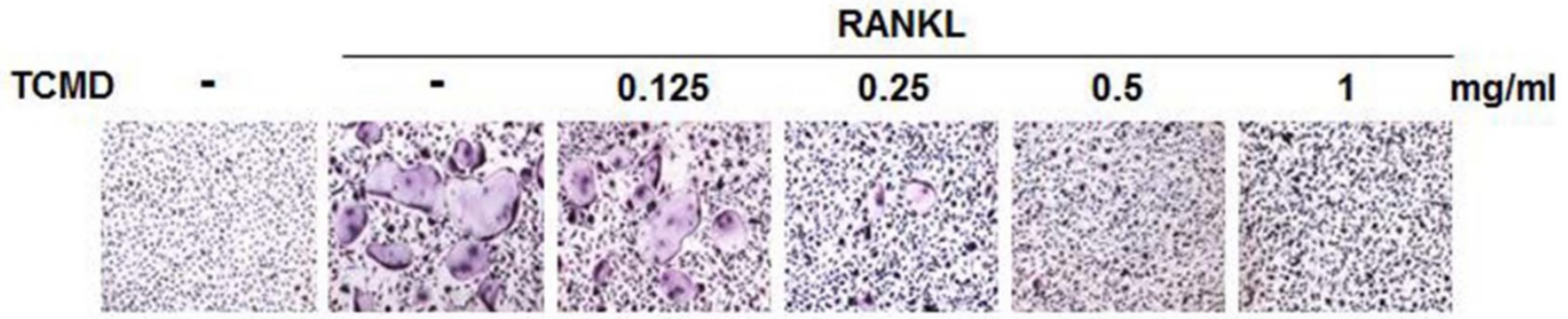

b

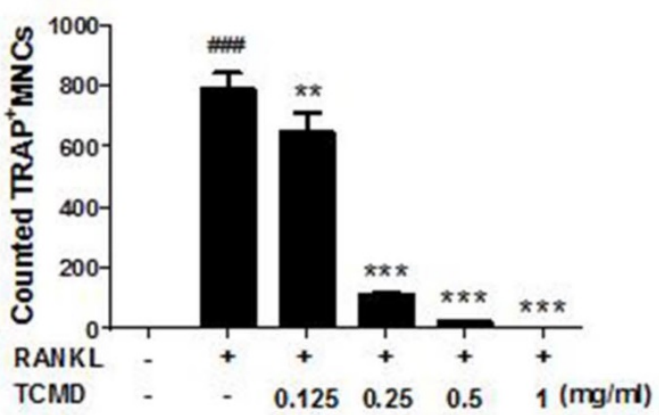

Figure 1. TCMD suppresses RANKL-induced osteoclast differentiation in BMDMs. (A) BMDMs were treated with M-CSF ( 30 ng/ml) and RANKL (100 $\mathrm{ng} / \mathrm{ml}$ ) for four days at the indicated concentration of TCMD. Multinucleated cells were visualized by TRAP staining. (B) TRAP-positive multinucleated cells were counted to determine osteoclast numbers. The results are expressed as mean \pm SD. $p<0.001$ vs. control , **p $<0.01$, $* * * p<0.001$ vs. RANKL group. 


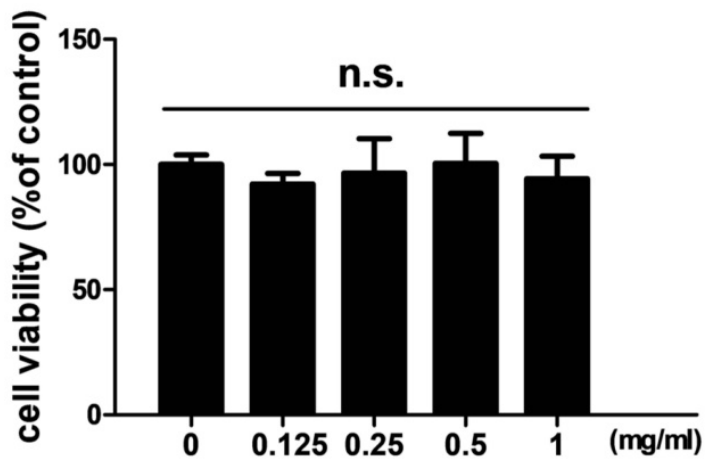

Figure 2. Effect of TCMD extract on BMDMs. BMDMs were cultured on a 96 -well plate for $24 \mathrm{~h}$ in the presence of M-CSF and treated with the indicated concentration of TCMD. Cell viability was determined as described in the Materials and Methods. The results are expressed as mean \pm SD.

a

RANKL

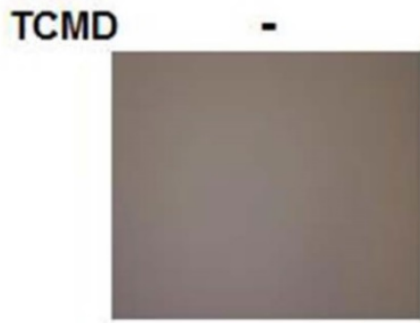

b

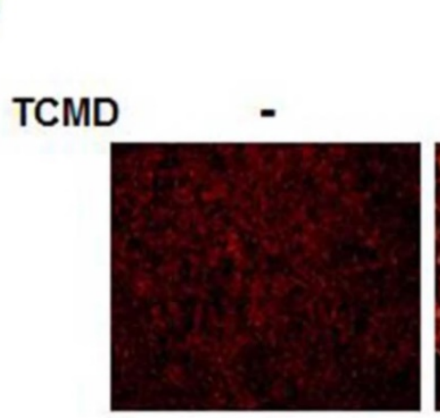

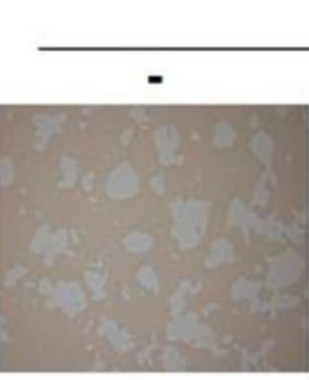

$0.5 \quad \mathrm{mg} / \mathrm{ml}$

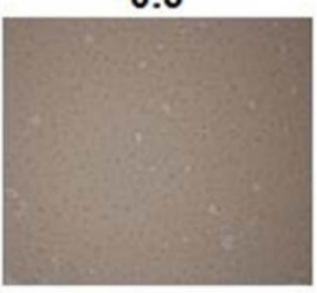

RANKL

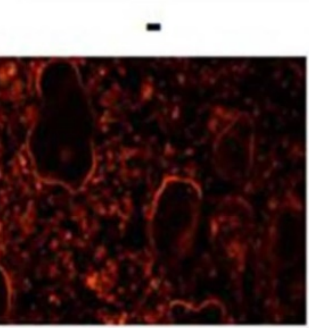

0.5

$\mathrm{mg} / \mathrm{ml}$

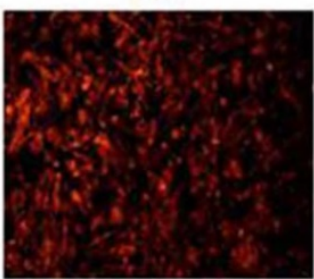

Figure 3. TCMD inhibits F-actin ring formation and osteoclastic bone resorption. (A) BMDMs were seeded on Osteo Assay Surface Plates and pretreated with TCMD $(0.5 \mathrm{mg} / \mathrm{ml})$ for $1 \mathrm{~h}$, then the cells were cultured with M-CSF $(30 \mathrm{ng} / \mathrm{ml})$ and RANKL (100 ng/ml) for an additional four days. (B) BMDMs were incubated with M-CSF $(30 \mathrm{ng} / \mathrm{ml})$ and RANKL $(100 \mathrm{ng} / \mathrm{ml})$ in the presence or absence of TCMD $(0.5 \mathrm{mg} / \mathrm{ml})$ for four days. The cells were fixed and stained for F-actin ring with Alexa-Fluor 594 phalloidin and imaged on a confocal system. Pit area was obtained based on the percentage of RANKL-induced destructive area obtained using image J program. Data are presented as mean \pm SD. ${ }^{\ldots \# p}<0.001$ vs. control; ***p $<0.001$ vs. RANKL group.

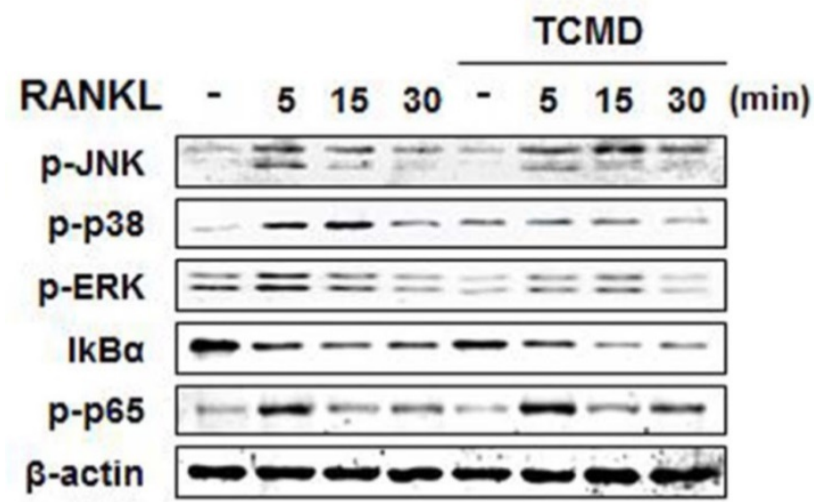

Figure 4. TCMD blocks RANKL-induced activation of p38 and ERK. BMDMs were pretreated with or without TCMD (0.5 mg/ml) for $1 \mathrm{~h}$ and then treated with RANKL $(100 \mathrm{ng} / \mathrm{ml})$ for the indicated times. Whole cell lysates were analyzed by Western blot with the indicated antibodies. The uncropped Western blots are presented in Supplementary Figure S1. 

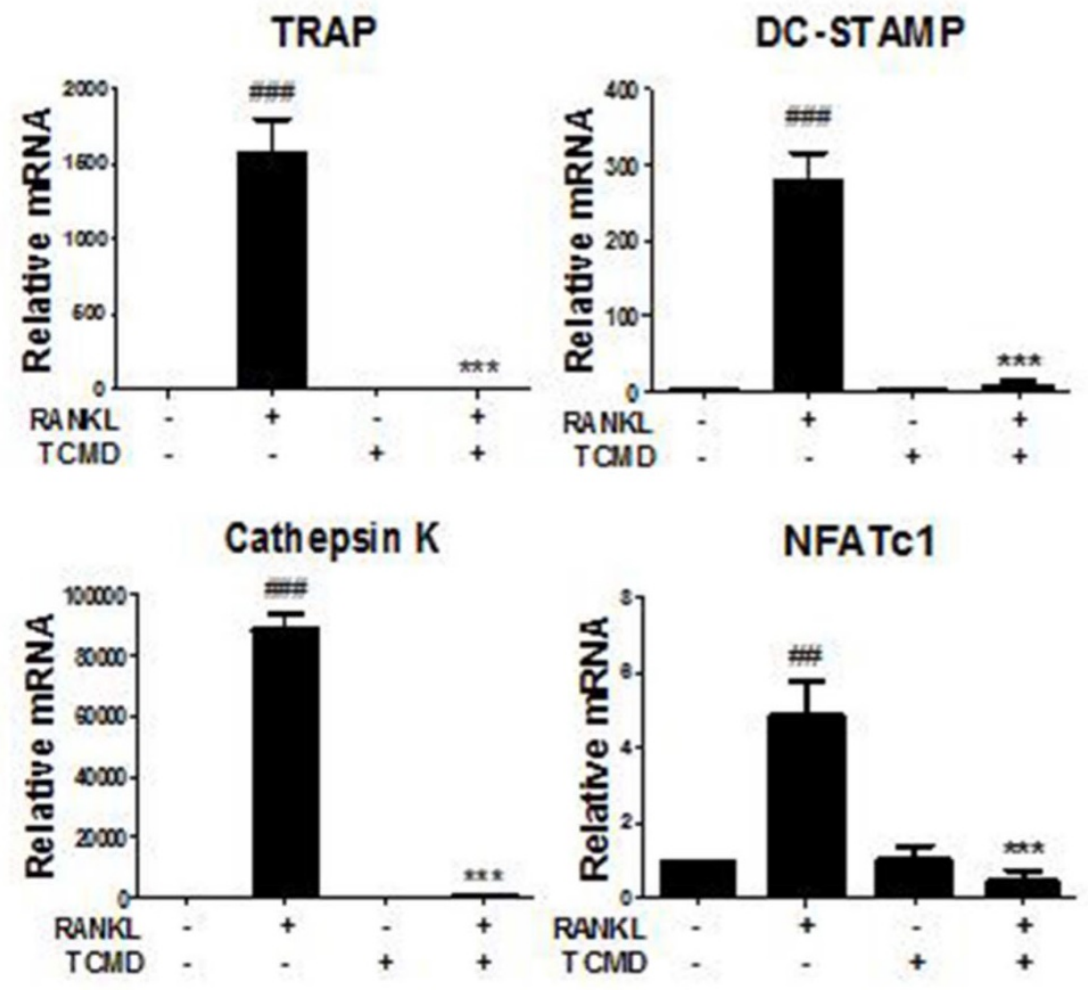

Figure 5. TCMD regulates RANKL-mediated osteoclast-specific gene expression. BMDMs were pretreated with or without TCMD (0.5 mg/ml) for $1 \mathrm{~h}$ and then treated with RANKL $(100 \mathrm{ng} / \mathrm{ml})$ for four days. Osteoclast-specific gene expression was analyzed using real-time PCR and results were normalized to the expression of $\beta$-actin. $p<0.01, \ldots+0.001$ vs. control, $* * * p<0.001$ vs. RANKL-treated group.

\section{Discussion}

Excessive osteoclast activity causes many diseases, and to date, anti-osteoporosis therapy has targeted this cell [18]. Therefore, inhibiting the formation of bone-resorbing osteoclasts through the suppression of RANKL signaling or its downstream pathways should be a rational target for the treatment of osteopenic diseases like osteoporosis. The pumpkin plant (Curcubita sp.) has long been used in traditional Asian medicine. This experiment is a new report investigating the anti-osteoclastogenesis effects of pumpkin tendril extract.

In the present study, we demonstrated for the first time that the water extract of TCMD, tendril of Cucurbita Moschata Duch., exerted an inhibitory effect on RANKL-induced osteoclastogenesis. BMDMs are osteoclast precursor cells that differentiate into osteoclasts in response to RANKL, which is expressed in osteoblasts, bone and lymphoid tissues, and is also a critical factor in osteoclastogenesis [19]. RANK/ RANKL interaction forms a multiple intracellular cytokine system that regulates osteoclast differentiation [20]. In our study, TRAP activities were measured in RANKL-induced BMDMs in order to evaluate the effects of TCMD on osteoclast differentiation. RANKL induced the development of multinucleated osteoclasts from precursors, and TCMD treatments exerted preventive effects on the formation of the TRAP-positive osteoclast without any cytotoxicity, as well as actin ring formation and bone resorption. Our previous study reported that rutin was abundant in the extract of TCMD, which had an anti-inflammatory effect [21]. Additionally, a recent study indicated that rutin had preventive effects against RANKL-induced osteoclast differentiation by inhibiting ROS production [22]. This is likely because TCMD contains rutin, resulting in a stronger inhibitory effect on osteoclast differentiation.

The connection between RANK and RANKL induces the recruitment and activation of TRAF6, which can regulate NF-kB and MAPKs signaling pathways [23]. NF-KB regulates osteoclast formation and activetion in bone resorption during RANKLinduced osteoclast signaling [24]. RANKL-induced NF- $\mathrm{KB}$ activation leads to the degradation of ІкBa, followed by nuclear translocation of the NF-kB subunit p65 and binding with DNA target sites [25]. In addition, it has been well established that the inhibition of MAPKs can suppress RANKL-induced osteoclast formation. RANKL-induced p38 and JNK phosphorylation is necessary for osteoclast differentiation signaling in BMDM $[26,27]$. However, ERK activation is more responsible for osteoclast survival than osteoclast differentiation [28]. In the present study, we found that TCMD inhibited 
osteoclast differentiation by suppressing RANKLmediated p38 and ERK phosphorylation and observed no evidence that TCMD could inhibit RANKL-induced JNK and p65 phosphorylation during osteoclastogenesis. However, the detailed mechanism by which TCMD suppresses RANKLinduced activation of MAPKs remains to be elucidated.

Activated MAPKs stimulate a variety of downstream targets including c-Fos and NFATc1, essential for the regulation of osteoclastogenesis in response to M-CSF and RANKL [29]. In particular, the amplification of NFATc1, a master regulator of osteoclast differentiation, regulates osteoclast-related genes such as cathepsin K, TRAP [30, 31]. DC-STAMP is an important regulator of cell fusion, and the fusion of mononuclear cells in bone resorption triggers the formation of multinucleated osteoclasts [32]. In this study, the expressions of all genes related to osteoclastogenesis, including TRAP, DC-STAMP, cathepsin K, and NFATc1, were significantly down-regulated by TCMD treatment. Consequently, TCMD may exert anti-osteoporotic effects by inhibiting the expression of the genes involved in osteoclastogenesis.

\section{Conclusions}

In summary, this is the first study to report that TCMD efficiently suppresses RANKL-induced osteoclast differentiation and bone-resorbing activity in vitro. Regarding molecular mechanisms, we showed that TCMD inhibits the RANKL-induced activation of p38 and ERK and down-regulates the expression of osteoclast-specific genes. Our results suggest that TCMD may be an effective traditional therapeutic medicine for the treatment of bone diseases. Further study is recommended to investigate the potential clinical application of TCMD in postmenopausal women with osteoporosis.

\section{Supplementary Material}

Supplementary figure S1.

http://www.medsci.org/v17p0632s1.pdf

\section{Acknowledgements}

This study was supported by a grant from the National Research Foundation (NRF, Korea-2017 R1D1A1A02019106), the Technology development Program of the Ministry of SMEs and Startups (MSS, Korea-C0396019) and the Korea institute for Advancement of Technology (KIAT) through the Encouragement Program for the Industries of Economic Cooperation Region (P0008590).

\section{Competing Interests}

The authors have declared that no competing interest exists.

\section{References}

1. Hadjidakis DJ, Androulakis II. Bone remodeling. Ann N Y Acad Sci. 2006; 1092: 385-96.

2. Boyle WJ, Simonet WS, Lacey DL. Osteoclast differentiation and activation. Nature. 2003; 423: 337-42

3. Fumimoto R, Sakai E, Yamaguchi Y, Sakamoto H, Fukuma Y, Nishishita K, et al. The coffee diterpene kahweol prevents osteoclastogenesis via impairment of NFATc1 expression and blocking of Erk phosphorylation. J Pharmacol Sci. 2012; 118: 479-86.

4. Kong YY, Yoshida H, Sarosi I, Tan HL, Timms E, Capparelli C, et al. OPGL is a key regulator of osteoclastogenesis, lymphocyte development and lymph-node organogenesis. Nature. 1999; 397: 315-23.

5. Yasuda H, Shima N, Nakagawa N, Yamaguchi K, Kinosaki M, Mochizuki S, et al. Osteoclast differentiation factor is a ligand for osteoprotegerin/osteoclastogenesis-inhibitory factor and is identical to TRANCE/RANKL. Proc Natl Acad Sci U S A. 1998; 95: 3597-602.

6. Takayanagi H. The role of NFAT in osteoclast formation. Ann N Y Acad Sci. 2007; 1116: 227-37.

7. Gohda J, Akiyama T, Koga $\mathrm{T}$, Takayanagi $\mathrm{H}$, Tanaka S, Inoue J. RANK-mediated amplification of TRAF6 signaling leads to NFATc1 induction during osteoclastogenesis. EMBO J. 2005; 24: 790-9.

8. Armstrong $\mathrm{AP}$, Tometsko ME, Glaccum $\mathrm{M}$, Sutherland $\mathrm{CL}$, Cosman $\mathrm{D}$, Dougall WC. A RANK/TRAF6-dependent signal transduction pathway is essential for osteoclast cytoskeletal organization and resorptive function. J Biol Chem. 2002; 277: 44347-56.

9. Lestari B, Meiyanto E. A Review: The Emerging Nutraceutical Potential of Pumpkin Seeds. I J C C. 2018; 9: 92-101.

10. Hossain MA, Lee SJ, Park NH, Birhanu BT, Mechesso AF, Park JY, et al. Enhancement of Lipid Metabolism and Hepatic Stability in Fat-Induced Obese Mice by Fermented Cucurbita moschata Extract. Evid Based complementary Alternat Med. : eCAM. 2018; 2018: 3908453.

11. Lestari B, Walidah Z, Utomo RY, Murwanti R, Meiyanto E. Supplementation with extract of pumpkin seeds exerts estrogenic effects upon the uterine, serum lipids, mammary glands, and bone density in ovariectomized rats. Phytother Res. PTR. 2019.

12. Kim HY, Nam SY, Yang SY, Kim HM, Jeong HJ. Cucurbita moschata Duch. and its active component, beta-carotene effectively promote the immune responses through the activation of splenocytes and macrophages. Immunopharmacol immunotoxicol. 2016; 38: 319-26.

13. Stevenson DG, Eller FJ, Wang L, Jane JL, Wang T, Inglett GE. Oil and tocopherol content and composition of pumpkin seed oil in 12 cultivars. J Agric Food Chem. 2007; 55: 4005-13.

14. Lee W, Ko KR, Kim HK, Lee DS, Nam IJ, Lim S, et al. Dehydrodiconiferyl Alcohol Inhibits Osteoclast Differentiation and Ovariectomy-Induced Bone Loss through Acting as an Estrogen Receptor Agonist. J Nat Prod. 2018; 81: 1343-56.

15. Lee JE, Shin HH, Lee EA, Van Phan T, Choi HS. Stimulation of osteoclastogenesis by enhanced levels of MIP-1alpha in BALB/c mice in vitro. Exp Hematol. 2007; 35: 1100-8.

16. Yamasaki T, Ariyoshi W, Okinaga T, Adachi Y, Hosokawa R, Mochizuki S, et al. The dectin 1 agonist curdlan regulates osteoclastogenesis by inhibiting nuclear factor of activated T cells cytoplasmic 1 (NFATc1) through Syk kinase. J Biol Chem. 2014; 289: 19191-203.

17. Wada T, Nakashima T, Hiroshi N, Penninger JM. RANKL-RANK signaling in osteoclastogenesis and bone disease. Trends Mol Med. 2006; 12: 17-25.

18. Teitelbaum SL. Bone resorption by osteoclasts. Science (New York, NY). 2000; 289: 1504-8.

19. Sims NA, Walsh NC. Intercellular cross-talk among bone cells: new factors and pathways. Curr Osteoporos Rep. 2012; 10: 109-17.

20. Zaidi M, Blair HC, Moonga BS, Abe E, Huang CL. Osteoclastogenesis, bone resorption, and osteoclast-based therapeutics. Journal of bone and mineral research : J Bone Miner Res. 2003; 18: 599-609.

21. Jeong HN, Choi JH, Lee HN, Lee SH, Cho SC, Park JH, et al. Inflammation inhibitory effect of water extract from pumpkin's tendril. Korean J Food Preserv. 2017; 24: 1122-28.

22. Kyung TW, Lee JE Shin $\mathrm{HH}$, Choi HS. Rutin inhibits osteoclast formation by decreasing reactive oxygen species and TNF-alpha by inhibiting activation of NF-kappaB. Exp Mol Med. 2008; 40: 52-8.

23. Leibbrandt A, Penninger JM. RANK/RANKL: regulators of immune responses and bone physiology. Ann N Y Acad Sci. 2008; 1143: 123-50.

24. Soysa NS, Alles N. NF-kappaB functions in osteoclasts. Biochem Biophys Res Commun. 2009; 378: 1-5.

25. Asagiri $M$, Sato $K$, Usami $T$, Ochi $S$, Nishina $H$, Yoshida $H$, et al Autoamplification of NFATc1 expression determines its essential role in bone homeostasis. J Exp Med. 2005; 202: 1261-9. 
26. Li X, Udagawa N, Itoh $\mathrm{K}$, Suda $\mathrm{K}$, Murase $\mathrm{Y}$, Nishihara $\mathrm{T}$, et al. p38 MAPK-mediated signals are required for inducing osteoclast differentiation but not for osteoclast function. Endocrinol. 2002; 143: 3105-13.

27. David JP, Sabapathy K, Hoffmann O, Idarraga MH, Wagner EF. JNK1 modulates osteoclastogenesis through both c-Jun phosphorylation-dependent and -independent mechanisms. J Cell Sci. 2002; 115: 4317-25.

28. Shim KS, Ma JY. Pine needles attenuate receptor activator for nuclear factor-B ligand (RANKL)-induced trabecular bone loss by inhibiting osteoclast differentiation. Integr Med Res. 2018; 7: 374-80.

29. Matsuo K, Irie N. [Transcription factors in osteoclast differentiation]. Nihon rinsho. 2005; 63: 1541-6.

30. Kwak HB, Lee BK, Oh J, Yeon JT, Choi SW, Cho HJ, et al. Inhibition of osteoclast differentiation and bone resorption by rotenone, through down-regulation of RANKL-induced c-Fos and NFATc1 expression. Bone. 2010; 46: 724-31.

31. Kim JH, Kim N. Regulation of NFATc1 in Osteoclast Differentiation. J Bone Metab. 2014; 21: 233-41.

32. Yagi M, Miyamoto T, Sawatani Y, Iwamoto K, Hosogane N, Fujita N, et al. DC-STAMP is essential for cell-cell fusion in osteoclasts and foreign body giant cells. J Exp Med. 2005; 202: 345-51. 